Western University

Scholarship@Western

Physiology and Pharmacology Publications

Physiology and Pharmacology Department

2013

\title{
Acute Vibration Induces Transient Expression of Anabolic Genes in the Murine Intervertebral Disc
}

\author{
Matthew R. McCann \\ Western University, mmccann4@uwo.ca \\ Priya Patel \\ Western University \\ Kim L. Beaucage \\ Western University \\ Yizhi Xiao \\ Western University \\ Corey Bacher \\ Western University \\ See next page for additional authors
}

Follow this and additional works at: https://ir.lib.uwo.ca/physpharmpub

Part of the Medical Physiology Commons, and the Pharmacy and Pharmaceutical Sciences Commons

Citation of this paper:

McCann, Matthew R.; Patel, Priya; Beaucage, Kim L.; Xiao, Yizhi; Bacher, Corey; Siqueira, Walter L.; Holdsworth, David W.; Dixon, S. Jeffrey; and Séguin, Cheryle A., "Acute Vibration Induces Transient Expression of Anabolic Genes in the Murine Intervertebral Disc" (2013). Physiology and Pharmacology Publications. 82.

https://ir.lib.uwo.ca/physpharmpub/82 
Authors

Matthew R. McCann, Priya Patel, Kim L. Beaucage, Yizhi Xiao, Corey Bacher, Walter L. Siqueira, David W. Holdsworth, S. Jeffrey Dixon, and Cheryle A. Séguin 
Supplementary Table 1

NCBI Gene Symbol

\begin{tabular}{|c|c|}
\hline $\begin{array}{l}\text { HPRT Fwd* } \\
\text { HPRT Rev* }\end{array}$ & $\begin{array}{l}\text { CAGGCCAGACTTTGTTGGAT } \\
\text { TTGCGCTCATCTTAGGCTTT }\end{array}$ \\
\hline Adamts4 Fwd* & GAGGAGGAGATCGTGTTTCCAG \\
\hline Adamts4 Rev* & CAAACCCTCTACCTGCACCC \\
\hline Adamts5 Fwd* & GGAGCGAGGCCATTTACAAC \\
\hline Adamts5 Rev* & GCGTAGACAAGGTAGCCCACTTT \\
\hline Acan Fwd* & CTGGGATCTACCGCTGTGAAG \\
\hline Acan Rev* & GTGTGGAAATAGCTCTGTAGTGGAA \\
\hline $\mathrm{Ben} \mathrm{Fwd}^{+}$ & ACGAATCCATGACAACCGTATC \\
\hline $\mathrm{Ben} \mathrm{Rev}^{+}$ & GCTCCTGGTTCAAAGCCACT \\
\hline Collal Fwd* & CTGGCGGTTCAGGTCCAAT \\
\hline Collal Rev** & TCCAGGCAATCCAGGAGC \\
\hline Col2al Fwd* & GCACATCTGGTTTGGAGAGACC \\
\hline Col2al Rev** & TAGCGGTGTTGGGAGCCA \\
\hline Ctgf Fwd* & GGGCCTCTTCTGCGATTTC \\
\hline Ctgf Rev* & ATCCAGGCAAGTGCATTGGTA \\
\hline Den Fwd $^{\dagger}$ & TCTTGGGCTGGACCATTTGAA \\
\hline Den $\operatorname{Rev}^{\dagger}$ & CATCGGTAGGGGCACATAGA \\
\hline Hifl $\alpha$ Fwd* & ACCTTCATCGGAAACTCCAAAG \\
\hline Hifl $\alpha \mathrm{Rev}^{*}$ & CTGTTAGGCTGGGAAAAGTTA \\
\hline Mmp3 Fwd* & TTGTCCCGTTTCCATCTCTCTC \\
\hline Mmp3 Rev* & TTGGTGATGTCTCAGGTTCCAG \\
\hline Mmp7 Fwd* & GCCAGGGAACACTCTAGGTCAT \\
\hline Mmp7 Rev'* & CTGCGTCCTCACCATCAGTC \\
\hline Mmp8 Fwd* & GATCTTCCTCCACACACAGCTTG \\
\hline Mmp8 Rev* & CTGCAACCATCGTGGCATT \\
\hline Mmp12 Fwd* & GCTTACCCCAAGCTGATTTCC \\
\hline Mmp12 Rev* & ATGTTTTGGTGACACGACGGA \\
\hline Mmp13 Fwd* & CTTCTTCTTGTTGAGCTGGAACTC \\
\hline Mmp13 Rev* & СTCTGTGGACCTCACTGTAGACT \\
\hline Timpl Fwd* & CTTGGTTCCCTGGCGTACTC \\
\hline Timp1 Rev* & ACCTGATCCGTCCACAAACAG \\
\hline Sox6 Fwd* & GGTCATGTTTCCCACCCACA \\
\hline Sox6 Rev* & TTCAGAGGGGTCCAAATTCCT \\
\hline Sox9 Fwd* & AGTACCCGCATCTGCACAAC \\
\hline Sox9 Rev* & TACTTGTAATCGGGGTGGTCTT \\
\hline Vcan Fwd & TTTTACCCGAGTTACCAGACTC \\
\hline Vcan $\operatorname{Rev}^{\dagger}$ & GGAGTAGTTGTTACATCCGTTG \\
\hline
\end{tabular}

Primer Sequence 5' $\rightarrow$ 3,

CAGGCCAGACTTTGTTGGAT

ThGGCTCATCTTAGGCTTT

GAGGAGGAGATCGTGTTTCCAG

CTGGGATCTACCGCTGTGAAG

TCCAGGCAATCCAGGAGC

GCACATCTGGTTTGGAGAGACC

GGGCCTCTTCTGCGATTTC

TCTTGGGCTGGACCATTT

GCCAGGGAACACTCTAGGTCAT

CTGCGTCCTCACCATCAGTC

GATCTTCCTCCACACACAGCTTG

GCTTACCCCAAGCTGATTTCC

CTTCTTCTTGTTGAGCTGGAACTC

AGTACCCGCATCTGCACAAC

TACTTGTAATCGGGGTGGTCTT

GGAGTAGTTGTTACATCCGTTG

Annealing Temperatures: ${ }^{*} 60^{\circ} \mathrm{C}$ degree; $+=58^{\circ} \mathrm{C}$ degrees; $\dagger=56^{\circ} \mathrm{C}$ degrees 